\title{
ASSOCIAÇÃO ENTRE ESTRESSE, NÍVEIS DE CORTISOL SALIVAR E LÍQUEN PLANO BUCAL: UM ESTUDO DE CASO-CONTROLE
}

\section{Lísia Daltro Borges Alves; Alessandra Lais Pinho Valente Pires²; Joana Dourado Martins; Juliana Araújo da Silva Simoura ${ }^{4}$; Eduardo Costa das Mercês ${ }^{5}$; Franco Arsati ${ }^{6}$; Ynara Bosco de Oliveira Lima Arsati ${ }^{7}$; Valéria Souza Freitas ${ }^{8}$.}

1. Bolsista PROBIC, Graduanda em Odontologia, Universidade Estadual de Feira de Santana, e-mail: lisia_94@hotmail.com

2. Aluna de Doutorado do Programa de Pós-graduação em Saúde Coletiva, Universidade Estadual de Feira de Santana, e-mail: lecavalent@hotmail.com

3. Pesquisadora do Núcleo de Câncer Oral, Universidade Estadual de Feira de Santana, e-mail: martinsjoana_1@hotmail.com

4. Pesquisadora do Núcleo de Câncer Oral, Universidade Estadual de Feira de Santana, e-mail: julianasimoura.uefs@gmail.com

5. Bolsista PIBIC, Graduando em Odontologia, Universidade Estadual de Feira de Santana, e-mail: ecmerces@gmail.com

6. Pesquisador do Núcleo de Câncer Oral, Universidade Estadual de Feira de Santana, e-mail: farsati@gmail.com

7. Pesquisadora do Núcleo de Câncer Oral, Universidade Estadual de Feira de Santana, e-mail: ynaralima76@gmail.com

8. Orientador, Departamento de Saúde, Universidade Estadual de Feira de Santana, e-mail: valeria.souza.freitas@gmail.com

PALAVRAS-CHAVE: líquen plano bucal; cortisol salivar; estresse.

\section{INTRODUÇÃO}

O Líquen Plano Bucal (LPB) é uma doença inflamatória crônica, com prevalência entre 0,1 a $4 \%$, dependendo da população estudada, que acomete principalmente mulheres, da raça/cor branca, a partir da quarta década de vida (BERMEJO-FENOLL; LOPEZ-JORNET, 2006; SCATTARELLA et al., 2011; PAYERAS et al., 2013).

Embora tenha sido descrita desde 1969, a etiologia do LPB ainda permanece incerta (ISMAIL; KUMAR; ZAIN, 2007). Na tentativa de compreender os possíveis fatores que desencadeiam ou agravam o LPB, os estudos têm investigado, sobretudo, o papel dos fatores psicológicos (estresse, ansiedade e depressão) nesta relação (SOUSA; ROSA, 2008; TAWIL; SEDIKI N; HASSAN H, 2009; SCATTARELLA et al., 2011).

Algumas pesquisas têm utilizado testes psicológicos com o objetivo de mensurar e quantificar esses fatores (SOUSA; ROSA, 2008). No entanto, devido à subjetividade destes testes, estudos atuais têm investigado sobre os biomarcadores salivares para a mensuração do estresse (NADENDLA, 2014; PIPPI et al., 2014; SHAH; ASHOK; SUJATHA, 2015). A saliva demonstra-se como um fluído biológico fundamental para o diagnóstico de doenças sistêmicas, avaliação de condições fisiológicas ou de situações capazes de induzir estados de estresse, ansiedade e depressão (THOMAS; HERTZMAN; POWER, 2009; CURCIO et al., 2013; GRYNDERUP et al., 2013).

Biomarcadores salivares como a desidroepiandrosterona, o cortisol e a alfa-amilase, estão sendo utilizados em pesquisas que buscam associar o LPB à fatores psicológicos (GIRARDI et al., 2011). Entretanto, estes estudos ainda são escassos e apresentaram resultados controversos ( KORAY; ET AL., 2003; GIRARDI et al., 2011; NADENDLA, 2014; PIPPI et al., 2014; SHAH; ASHOK; SUJATHA, 2015). Deste modo, o objetivo deste trabalho foi avaliar a associação entre estresse e o LPB.

\section{MATERIAL E MÉTODOS}

Trata-se de um estudo caso-controle conduzido no Centro de Referência em Lesões Bucais (CRLB) da Universidade Estadual de Feira de Santana (UEFS), após aprovação do Comitê de Ética em Seres Humanos (CAAE: 05590612.7.0000.0053; parecer 114.132). O 
grupo caso foi constituído por 21 indivíduos com diagnóstico clínico/histopatológico de LPB, e o controle, por 21 indivíduos sem a doença pareado por sexo e idade. Os grupos foram submetidos a exame clínico da cavidade bucal, entrevista estruturada, teste psicológico (Escala de Estresse Percebido) e coleta de saliva em três momentos distintos. O padrão de secreção de cortisol salivar foi avaliado pelo método imunoenzimático. Utilizou-se o teste de MannWhitney para comparar os grupos com relação ao escore de estresse e o padrão de secreção do cortisol salivar e o Coeficiente de Correlação de Spearman, para avaliar a concentração de cortisol com o escore de estresse. O nível de significância foi de 5\%.

\section{RESULTADOS E DISCUSSÃO}

A população do estudo foi constituída por 42 indivíduos, em que 69,0\% apresentavam idade acima dos 40 anos, com média de 48,64 anos, estando os valores mínimos e máximo variando de 25 a 80 anos. A maioria dos indivíduos era mulher (71,4\%); auto declarava-se não brancos $(85,71 \%)$, com uma relação conjugal estável, ou seja, casados/união consensual (59,49\%). A análise da situação de trabalho demonstra que $73,8 \%$ tinham uma renda familiar superior a dois salários mínimos e, 59,52\%, estavam ocupacionalmente ativos. Quanto aos aspectos clínicos do LPB, as lesões brancas (reticular e em placa) foram mais predominantes $(85,71 \%)$ e, as vermelhas (atrófica e erosiva), apresentaram-se em 14,29\% dos indivíduos.

Ao aplicar a Escala de Estresse Percebido, os escores de estresse variaram de 0 a 41, com mediana de 26(15-26) no grupo caso e de 17(12-17) nos controles, com diferença estatisticamente significante entre os grupos ( $\mathrm{p}=0,026$; Mann-Whitney). Os escores de estresse percebido foram categorizados, observando-se associação estatisticamente significativa $(\mathrm{OR}=4,0[1,11-14,80])$ (Tabela 1).

Tabela 1. Distribuição dos indivíduos dos grupos caso e controle, odds ratio (OR), intervalo de confiança (IC 95\%), quanto à presença de estresse, em Feira de Santana, 2014-2015.

\begin{tabular}{lcccccc}
\hline & \multicolumn{2}{c}{ CASOS } & \multicolumn{2}{c}{ CONTROLES } & \\
ESTRESSE PERCEBIDO & $\mathrm{n}(=21)$ & $\%$ & $\mathrm{n}(=21)$ & $\%$ & OR & IC (95\%) \\
\hline Presença de estresse percebido & 15 & 71,42 & 6 & 39,13 & \multirow{2}{*}{4} & \multirow{2}{*}{$1,11-14,80$} \\
Ausência de estresse percebido & 8 & 38,08 & 13 & 60,87 & & \\
\hline
\end{tabular}

*Teste $\mathrm{X}^{2}$ de McNemar

LUNDQVIST et al., (2006) também verificaram além de ansiedade e depressão, uma forte correlação entre LPB e estresse percebido. Entretanto, GIRARDI et al., (2011), em um estudo de caso-controle, os valores não diferiam entre os grupos.

A coleta de saliva para a análise do cortisol salivar foi realizada em três momentos no mesmo dia, de modo a permitir a avaliação da variação do padrão de secreção deste hormônio ao longo do dia. As medianas das concentrações salivares de cortisol ao acordar e trinta minutos depois de acordar foram de $0,28 \mu \mathrm{g} / \mathrm{dL}(0,22-0,28), 0,28 \mu \mathrm{g} / \mathrm{dL}(0,22-0,28)$ no grupo-caso e de $0,29 \mathrm{n} \mu \mathrm{g} / \mathrm{dL}(0,21-0,29), 0,35 \mu \mathrm{g} / \mathrm{dL}(0,21-0,35)$ no grupo-controle respectivamente. Nas amostras salivares coletadas à noite, as medianas das concentrações de cortisol foram de 0,05 $\mu \mathrm{g} / \mathrm{dL}(0,03-0,05)$ nos indivíduos com LPB e de $0,05 \mu \mathrm{g} / \mathrm{dL}(0,02-0,05)$ nos controles (Figura $1)$.

Figura 1. Boxplot da concentração de cortisol $(\mu \mathrm{g} / \mathrm{dL})$ ao acordar, trinta minutos depois de acordar e antes de dormir dos indivíduos atendidos nas clínicas de Odontologia em Feira de Santana, 2014-2015.
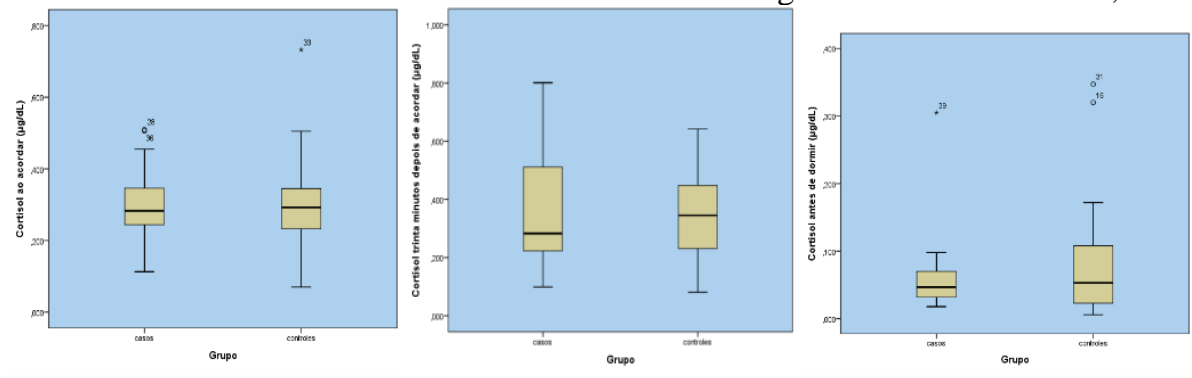
Quando realizado o teste de Friedman para verificar a variação dos valores da concentração de cortisol, constatou-se diferença significativa ao longo do dia $(p=0,00)$. $\mathrm{Na}$ Figura 2, têm-se os níveis médios do cortisol salivar nos diferentes horários de coleta.

Figura 2. Concentração de cortisol ao acordar, trinta minutos depois de acordar e antes de dormir dos indivíduos atendidos nas clínicas de Odontologia em Feira de Santana, 2014-2015.

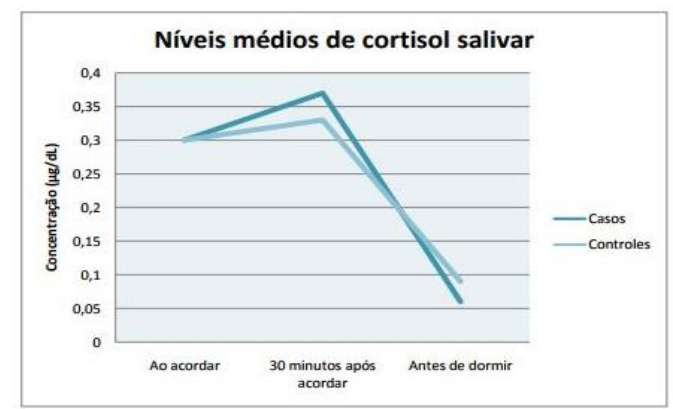

Figura 3. Correlação entre os valores de cortisol no turno matutino e o escore total do estresse percebido dos indivíduos investigados, em Feira de Santana, 2014-2015.

Ao comparar os grupos, não foi observada diferença significativa entre as concentrações ao acordar $(\mathrm{p}=0,98)$, trinta minutos depois de acordar $(\mathrm{p}=0,95)$ e noturno $(\mathrm{p}=0,97)$. Os valores de cortisol salivar de cada indivíduo, ao acordar e trinta minutos depois de acordar, foram transformados em Resposta do cortisol ao acordar (RCA). A média da RCA foi $0,08( \pm 0,25)$ $\mu \mathrm{g} / \mathrm{dL}$ para o grupo LPB, $0,02( \pm 0,17) \mu \mathrm{g} / \mathrm{dL}$ para o grupo controle. Quando feita a comparação entre os grupos (Teste $t)$, não houve diferença estatisticamente significante $(p=0,37)$. $O$ Coeficiente de Correlação de Spearman foi empregado para verificar a correlação entre as concentrações de cortisol com o escore de estresse. Observou-se correlação significativa entre as variáveis $(\mathrm{p}=0,04)$. Na Figura 3, observou-se uma ascendência positiva entre estas variáveis, o que denota uma tendência dos dados quanto à relação entre o aumento proporcional das concentrações de cortisol e o escore de estresse da população investigada.

Os resultados demonstraram uma associação estatisticamente significante no padrão de secreção de cortisol salivar ao longo do ciclo circadiano nos grupos, com um aumento do padrão de secreção do cortisol, em média de $23,3 \%$ nos casos e de $10 \%$ nos controles, entre o acordar e 30 minutos após, e com declínio no período noturno, seguindo o padrão descrito previamente por FEDERENKO, (2004) e HELLHAMMER; WÜST; KUDIELKA, (2009).

Não foi verificada diferença estatisticamente significativa da concentração deste hormônio entre os três tempos de coleta nos grupos avaliados. Além disso, quando se avaliou a RCA, não houve também diferença estatisticamente significativa entre os grupos. Nossos achados contrapõem os resultados de KORAY el al., (2003), que verificaram níveis de cortisol salivar significativamente superiores em indivíduos com LPB ao realizar apenas uma coleta durante o dia. SHAH; ASHOK; SUJATHA, (2015) reportam maiores níveis de cortisol, ansiedade, depressão e estresse no grupo com LPB.

No estudo de GIRARDI et al., (2011), as concentrações salivares de cortisol, assim como no nosso estudo, não diferiram entre os grupos, portanto, para estes autores o cortisol não deve ser empregado como biomarcador salivar associado a esta doença.

\section{CONSIDERAÇÕES FINAIS}

O presente estudo demonstrou uma associação significativa entre o estresse nos indivíduos com LPB, embora o padrão de secreção de cortisol salivar não diferiram entre os grupos. Os resultados devem contribuir para uma melhor compreensão do papel dos fatores psicológicos, em especial o estresse, em relação ao LPB, através de seus efeitos sobre a resposta imune. Os baixos níveis de cortisol salivar encontrado em indivíduos com LPB podem ser resultantes de alterações inflamatórias locais que culminam com aumento da atuação do eixo 
HHA. Tais achados indicam a necessidade de novas investigações, com diferentes biomarcadores, envolvendo outros eixos do sistema nervoso autônomo, que possam melhor refletir a resposta ao estresse nos indivíduos com esta lesão.

\section{REFERÊNCIAS}

BERMEJO-FENOLL, A.; LOPEZ-JORNET, P. Familial oral lichen planus: presentation of six families. Oral Surgery, Oral Medicine, Oral Pathology, Oral Radiology and Endodontology, v. 102, n. 2, p. 12-15, 2006.

CURCIO, W. B. et al. Nível de cortisol salivar entre crianças em tratamento odontológico - Um estudo piloto. Pesquisa Brasileira em Odontopediatria e Clinica Integrada, v. 13, n. 1, p. 510, 2013.

FEDERENKO, I. ET AL. Free cortisol awakening responses are influenced by awakening time. Psychoneuroendocrinology., v. 29, p. 174-84, 2004.

GAVIC, L. et al. The role of anxiety, depression, and psychological stress on the clinical status of recurrent aphthous stomatitis and oral lichen planus. Journal of oral pathology \& medicine: official publication of the International Association of Oral Pathologists and the American Academy of Oral Pathology, v. 43, n. 6, p. 410-7, 2014.

GIRARDI, C. et al. Salivary cortisol and dehydroepiandrosterone (DHEA) levels, psychological factors in patients with oral lichen planus. Archives of Oral Biology, v. 56, n. 9, p. 864-868, 2011.

GRYNDERUP, M. B. et al. A two-year follow-up study of salivary cortisol concentration and the risk of depression. Psychoneuroendocrinology, v. 38, n. 10, p. 2042-2050, 2013.

HELLHAMMER, D. H.; WÜST, S.; KUDIELKA, B. M. Salivary cortisol as a biomarker in stress research. Psychoneuroendocrinology, v. 34, n. 2, p. 163-171, 2009.

ISMAIL, S. B.; KUMAR, S. K. S.; ZAIN, R. B. Oral lichen planus and lichenoid reactions: etiopathogenesis, diagnosis, management and malignant transformation. Journal of oral science, v. 49, n. 2, p. 89-106, 2007.

KORAY, M.; ET AL. The evaluation of anxiety and salivary cortisol levels in patients with oral lichen planus. Oral diseases., v. 9, n. 6, p. 298-301, 2003.

LUNDQVIST, E.; ET AL. Psychological health in patients with genital and oral erosive lichen planus. Journal of the European Academy of Dermatology and Venereology, v. 20, n. 6, p. 661666, 2006.

NADENDLA, L. K. Association of Salivary Cortisol and Anxiety Levels in Lichen Planus Patients. Journal of Clinical and Diagnostic Research, p. 10-13, 2014.

PAYERAS, M. R. et al. Oral lichen planus: Focus on etiopathogenesis. Archives of Oral Biology, v. 58, n. 9, p. 1057-1069, 2013.

PIPPI, R. et al. Diurnal trajectories of salivary cortisol, salivary ??-amylase and psychological profiles in oral lichen planus patients. Journal of biological regulators and homeostatic agents, v. 28 , n. 1, p. 147-154, 2014.

SCATTARELLA, A. et al. Oral lichen planus and dental hygiene: A case report. International Journal of Dental Hygiene, v. 9, n. 2, p. 163-166, 2011.

SHAH, B.; ASHOK, L.; SUJATHA, G. P. Evaluation of salivary cortisol and psychological factors in patients with oral lichen planus. Indian J Dent Res., v. 20, n. 3, p. 288-292, 2015.

SOUSA, F. A. C. G. DE; ROSA, L. E. B. Oral lichen planus: clinical and histopathological considerations. Brazilian journal of otorhinolaryngology, v. 74, n. 2, p. 284-92, 2008.

TAWIL, E.; SEDIKI N; HASSAN H. Psychobiological Aspects of Patients with Lichen Planus. Current Psychiatry [Egypt]Current Psychiatry, v. 1616, n. 44, p. 370-80, 2009.

THOMAS, C.; HERTZMAN, C.; POWER, C. Night work, long working hours, psychosocial work stress and cortisol secretion in mid-life: evidence from a British birth cohort. Occupational and Environmental Medicine, v. 66, n. 12, p. 824-831, 2009. 